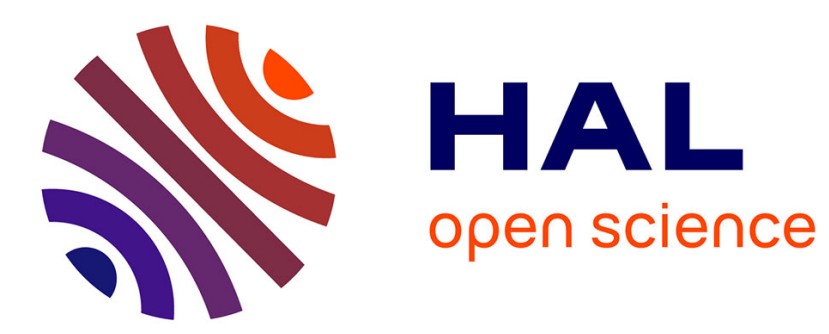

\title{
Simulated behaviour of field-assisted ionisation in the theory of Synthetic Poole Frenkel effect
}

\author{
A. Pillonnet, R. Ongaro
}

\section{To cite this version:}

A. Pillonnet, R. Ongaro. Simulated behaviour of field-assisted ionisation in the theory of Synthetic Poole Frenkel effect. Journal de Physique III, 1991, 1 (8), pp.1449-1454. 10.1051/jp3:1991202 . jpa00248670

\section{HAL Id: jpa-00248670 https://hal.science/jpa-00248670}

Submitted on 1 Jan 1991

HAL is a multi-disciplinary open access archive for the deposit and dissemination of scientific research documents, whether they are published or not. The documents may come from teaching and research institutions in France or abroad, or from public or private research centers.
L'archive ouverte pluridisciplinaire HAL, est destinée au dépôt et à la diffusion de documents scientifiques de niveau recherche, publiés ou non, émanant des établissements d'enseignement et de recherche français ou étrangers, des laboratoires publics ou privés. 


\title{
Simulated behaviour of field-assisted ionisation in the theory of Synthetic Poole Frenkel effect
}

\author{
A. Pillonnet and R. Ongaro \\ Université Claude Bernard Lyon I, Bât. 721, 43 Bld du 11 Novembre 1918, 69622 Villeurbanne \\ Cedex, France
}

(Received 28 December 1990, revised 18 March 1991, accepted 22 April 1991)

\begin{abstract}
Résumé. - Une simulation du comportement du courant permanent, en fonction du champ électrique, est réalisée dans le cadre d'une nouvelle approche des théories Poole et Poole-Frenkel (PF) que nous nommons effet Poole-Frenkel Synthétique (SPF) (Ongaro et Pillonnet, dans IEE Proc. PtA 138, 127-37). L'illustration quantitative montre que notre approche SPF réussit à joindre, en une formulation unique, les approches Poole et PF précédentes, qui apparaissent alors comme des cas limites. Cependant on souligne les difficultés qui peuvent être attendues en ce qui concerne la détermination des paramètres impliqués.
\end{abstract}

\begin{abstract}
A simulation is made of the behaviour of dc-current versus electric field when use is made of a new approach of Poole and Poole-Frenkel (PF) theories, we designated as Synthetic Poole Frenkel (SPF) effect (Ongaro and Pillonnet, in IEE Proc. PtA 138, 127-37). Quantitative illustration shows that our SPF approach succeeds fairly well in joining in a unique formulation the early Poole and PF approaches, which appear then as limiting cases. However, it is stressed that difficulties can be expected as regards the determination of the involved parameters.
\end{abstract}

\section{Introduction.}

A generalization of the theories of Poole and Poole-Frenkel (PF) field-assisted ionisation processes, which was designated as Synthetic Poole Frenkel (SPF) effect, was derived recently [1]. This consisted mainly of a derivation of the lowering of potential energy by the field of an assembly of Coulomb potential wells, and joining in a unique formulation Poole and Poole Frenkel theories; as is well-known, the latter took account of potential interactions while the former did not do so. The resulting potential lowering, $\Delta \Phi_{z}^{\prime}$, obtained in a model of interactions limited within $Z$ pairs of wells, was derived in a one-dimensional treatment. Only potential energy variations are accounted for, as this is by far the most usually adopted assumption. In other words, our SPF effect is not worked out on the approach of Mahapatra and Roy [2a] and Roy [2b, 2c]; these authors considered variations of total energy, and their results indicated that the ionisation potential predicted by PF theories gets lowered by a factor of 2.

In our approach quoted above [1], only theoretical developments were dealt with, and 
these were concerned uniquely with the derivation of barrier lowering. There remained to investigate the changes our theory was expected to introduce in the behaviour of current versus field. This is the purpose of the present paper, which brings, by means of computer simulations, an illustration of behaviours that can be expected. To do this, $\Delta \Phi_{z}^{\prime}$ obtained from SPF is introduced into a formulation of the probability of ionisation, previously established on the basis of Fermi-Dirac statistics [3].

\section{Variations of free electron densities according to SPF.}

The study of variations of free electron densities according to SPF can be conveniently carried out if we introduce a system of reduced variables which are discussed below.

2.1 REDUCED SPF EQUATIONS. - When an unspecified number $2 Z$ of potential wells are interacting, we showed that the reduced lowering of potential by the field, denoted in reference [1] as $\Delta S_{z}$, can be written as

$$
\Delta S_{z}=\frac{\Delta \Phi_{z}^{\prime}}{\Phi^{\prime}}=\sum_{k=0}^{z-1} \frac{1}{2 k+1} \frac{1}{1-\frac{\xi_{z}^{\prime 2}}{(2 k+1)^{2}}}+\frac{1}{2} f\left(1+\xi_{z}^{\prime}\right)-\Sigma_{z}
$$

The symbols in (1) have meanings as given below: $\Phi^{\prime}=\beta^{2} / e a$ is the zero-field inner barrier lowering of an isolated pair of wells $(Z=1)$ due to potential overlap, with $\beta=$ $\left(e^{3} / \pi \varepsilon_{0} \varepsilon\right)^{1 / 2}$ and $a$ is the mean donor spacing. $f=F / F_{\mathrm{t}}$ is the reduced applied field, with $F_{\mathrm{t}}=(\beta / e a)^{2}$ standing for Vollmann's [4] transition field between the previously defined Poole and PF effects. $\xi_{z}^{\prime}(<0)$ is the reduced abscissa $\left(\xi_{z}^{\prime}=\left(x_{\mathrm{m}^{\prime}}\right)_{z} / \frac{a}{2}\right)$ of the potential maximum with a field applied, the origin being taken midway between two adjacent sites. $\Sigma_{z}=\sum_{k=0}^{z-1}(2 k+1)^{-1} \Delta \Phi_{z}^{\prime}$ is the notation used in reference [1] for the SPF equivalent of the usual PF barrier lowering $\Delta \Phi=\beta \sqrt{F}$.

An approximate expression for $\Delta S_{z}$ can also be used [1], as it fits fairly well in equation (1) over a large range of practical values of $F$. This approximate expression, $\Delta A_{z}$, is :

$$
\Delta A_{z}=\frac{f}{2+\sqrt{f}}+\frac{0.173 f^{143}}{1+0.5 f^{12}} .
$$

This could assist experimentalists in applying SPF theory to experimental data. Considering a model with only one donor level in the gap, and applying Fermi-Dirac statistics, it was shown [3] that the relative density of released electrons, in PF theory (isolated well), is

$$
n_{\mathrm{r}}=\frac{2 s \frac{q-1}{q}}{\left(1+\frac{s}{q} \mathrm{e}^{\eta-\alpha}\right)\left[1+\sqrt{1+4 s \frac{q-1}{q} \frac{\mathrm{e}^{\eta-\alpha}}{\left(1+\frac{s}{q} \mathrm{e}^{\eta-\alpha}\right)^{2}}}\right]}
$$

The symbols in (3) have meanings as given below : $n_{r}\left(=n / N_{\mathrm{c}}\right)$ is the density of free electrons relative to the density $N_{\mathrm{c}}$ of conduction band states; $s=N_{\mathrm{d}} / N_{\mathrm{c}}$ is the relative density of donors ; $q=N_{\mathrm{d}} / N_{\mathrm{a}}$ is a compensation rate expressed as the ratio of donor and deep acceptor 
densities ; $\eta=\Phi / k T$ is the dimensionless apparent donor depth ; $\alpha$ is either the dimensionless SPF field-induced potential lowering, $\Delta \Phi_{z}^{\prime} / k T$, or the usual dimensionless PF lowering, $\Delta \Phi / k T$.

2.2 VARIATIONS OF FREE ELECTRON DENSITIES WITH FIELD. - Simulations are performed by simply putting $\Delta \Phi_{z}^{\prime}$ from equation (1) into the variable $\alpha$ of equation (3), and by choosing appropriate sets of values for the parameters of this equation. For comparing with the case of PF effect developed in reference [3], simulation of equation (3) is also made with the same set of parameters and with the PF variable $\alpha=\beta \sqrt{F} / k T$.

Figure 1, plotted as $\log n$, versus $\sqrt{F}$, shows that SPF effect (full lines) displays the same

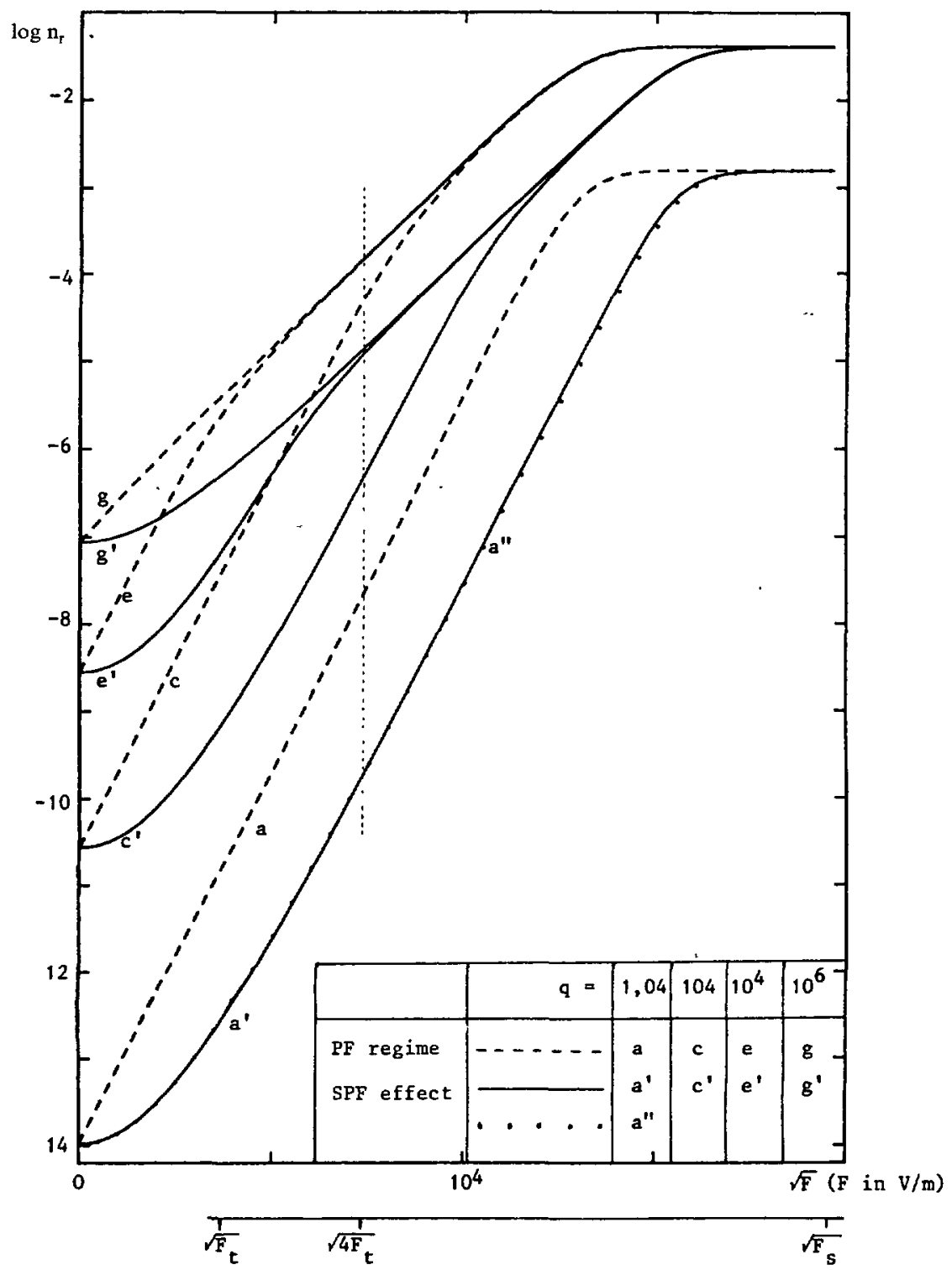

Fig. 1. - Variations of $\log n_{r}$ versus field for PF and SPF effects. $\varepsilon=2.2 ; a=140 \AA\left(N_{d} \sim\right.$ $\left.3.6 \times 10^{17} \mathrm{~cm}^{-3}\right) ; T=300 \mathrm{~K} ; s=0.041\left(N_{\mathrm{c}}=8.9 \times 10^{18} \mathrm{~cm}^{-3}\right) ; Z_{\mathrm{m}}=2 ; \Phi=1 \mathrm{eV} ; \Phi_{\mathrm{az}}^{\prime}=0.75 \mathrm{eV}$. 
high-field behaviour as PF effect (dashed lines). This corroborates the well-known statement of Dussel and Böer [5] that, for a given donor density, Coulombic wells become disconnected, i.e. their mutual interactions vanish, as the field reaches some threshold value. We marked on a second axis three remarkable points : the transition field $F_{t}$ of Vollmann ; another transition field $F_{\mathrm{t}}^{\prime}=4 F_{\mathrm{t}}$ which can be deduced from a paper by Hill [6] and which clearly seems more appropriate than Vollmann's (the small-dashed vertical line makes conspicuous that the near range of $F_{\mathrm{t}}^{\prime}$ is that for Poole to PF transition); $F_{\mathrm{s}}$ is the field of saturation $\left(F_{\mathrm{s}}=(\Phi / \beta)^{2}\right)$ beyond which donors are fully empty. Dots (a") marked on curve (a') result from the approximation of SPF effect by equation (2). For the sake of comparing significantly SPF and PF effects, equal depths must be chosen for donors in the two cases. However, if a depth $\Phi_{\mathrm{PF}}$ is assumed for the case of independent wells, it was shown in reference [1] that, when the wells are overlapping, an apparent depth

$$
\Phi_{a z}^{\prime}=\Phi-\Phi^{\prime} \Sigma_{z}
$$

must be substituted for $\Phi_{\mathrm{PF}}$ in equation (3). Therefore, the condition $\Phi_{\mathrm{PF}}=\Phi_{a z}^{\prime}$ was satisfied as follows. First $\Phi_{\mathrm{PF}}=0.75 \mathrm{eV}$; then it is assumed for the overlap case that the assembly of wells can be truncated at an energy $k T$ below the bottom of the conduction band, so that interactions of wells are limited to within an assembly of 4 wells $\left(Z=Z_{\mathrm{m}}=2\right)$. The actual depth $\Phi$ to be chosen in SPF is then $\Phi=1 \mathrm{eV}$.

In the low field range $(f<1)$, Poole regime manifests itself as a progressive smoothing of curves. It is remarkable that such a smoothing is in conformity with a lot of experimental curves of the literature. However, in addition to considering Poole regime as working preferentially at low field strengths, two other interpretations were proposed. One consisted of considering that carriers of another origin could coexist with the carriers released by the field (Hirai and Nakada [7]). Likewise, Tanaka and Inuishi [8] explain some curves of conductivity versus field, obtained on polyethylene films, by means of a model including both deep donors and shallow traps. This is in the right view of an analysis by Simmons [9]. The other model of interpretation consisted of explaining the shape of the low field conductivity by contriving a three-dimensional PF (3D-PF) effect (see for example Pillonnet [10]). For instance, Lovell [11] deemed that measurements on (epoxy) polymers were in good agreement with Hill's [6] 3D-PF approach, in which conductivity $(\sigma)$ is given by

$$
\sigma \propto k T \frac{\beta^{2}}{\alpha^{3}} \exp (-\Phi / k T)(\alpha \operatorname{ch} \alpha-\operatorname{sh} \alpha) .
$$

A critical comparative analysis of 3D-PF theories can be found in reference [10]. If instead, the low field smoothing is referred to Poole's law, an advantage of SPF theory could be that it combines the early Poole and PF theories in a unique formulation.

Comparison between PF and SPF behaviours as a whole shows that the latter is shifted downward by an amount of the order of $\frac{1}{2} \Phi^{\prime} \Sigma_{2}$ in the medium range, and that this quantity was found practically independent of $Z$. The less efficiency of the field at lower strengths arises from the fact that, in the usual theories, excluding the approach of Mahapatra and Roy $[2 a, b, c]$, a larger barrier lowering results from considering the wells as isolated. There is no need of commenting on both the unusual high-field PF and SPF saturation displayed by figure 1, and the existence of two slopes in cases of limited compensation, with slope parameters in a ratio of two ; this saturation was shown [3] to be derivable readily from FermiDirac statistics. 


\section{Conclusion.}

The present study allows to distinguish quantitatively the degree of resemblance or of dissimilarity, that can be expected between PF and SPF approaches. Also, it allows to verify that SPF theory could be considered as an improved synthesis of Poole and PF theories. Its more general character, as a method of approaching field-assisted electron thermal release, would rather plead in favour of this theory. However, it must be emphasized here, and from a practical point of view, that a quantitative access to the parameters associated with SPF could be difficult, for the following reasons.

(i) In a favourable case where saturation would appear, the limiting value of current could allow to accede to $N_{\mathrm{d}}-N_{\mathrm{a}}$, through equation (3). Now, saturation with the field cannot be expected easily to arise at high fields, because other phenomena like impact ionisation should be very likely in this range. Cases where the curves display two slopes or even one slope with a one-half slope parameter, are those where $N_{\mathrm{d}} \gg N_{\mathrm{a}}$.

(ii) Knowledge of $N_{\mathrm{d}}$ is needed to derive the mean intersite distance $a$, and to evaluate the number $Z_{\mathrm{m}}$ of pairs to which one can circumscribe the overlapping of wells. Therefore, it is difficult to determine the lowering of the intersite potential $\left(\Delta \Phi_{z}^{\prime}\right)$ by the field.

(iii) The apparent activation energy $\Phi_{a z}^{\prime}$ cannot be deduced by extrapoling a curve to the origin, contrary to the case of the earlier PF theories. We showed elsewhere [12] that, deriving it from Arrhenius plots cannot be considered as an undisputable means. Likewise, the actual depth $\Phi$ of the wells is difficult to obtain this way, so that other independent methods of determination are necessary.

(iv) Ordinate of curves at $f=0$ is associated with the compensation ratio $q$, in an involved manner. In a favourable case where saturation would appear, knowledge of $n_{r}(\infty)$ and $n_{r}(0)$ would yield

$$
\frac{n_{r}(\infty)}{n_{r}(0)}=\frac{1}{2}\left(1+\frac{N_{\mathrm{a}}}{N_{\mathrm{c}}} \mathrm{e}^{\eta}\right)\left[1+\sqrt{1+4 n_{r}(\infty)\left(\frac{\mathrm{e}^{\eta}}{\left(1+\frac{N_{\mathrm{a}}}{N_{\mathrm{c}}} \mathrm{e}^{\eta}\right)^{2}}\right)}\right.
$$

If $N_{\mathrm{a}} / N_{\mathrm{c}}$ can be known some other way, this equation determines $\Phi_{a z^{*}}^{\prime}$ However, this is convenient only in cases where a unique level is considered, and we know that this assumption cannot be derived safely from Arrhenius diagrams.

(v) Curves of figure 1 are drawn only in the simplified case where the screening of donors by the mobile charged entities, present in the material, can be neglected. However, when developping our SPF theory [1], we analysed carefully the theoretical consequences of considering wells sharper-than Coulombic wells. We showed there that the difficulties resulting from the non-convergence of sums like the one of equation (1) are tided over. However, a short analysis of the literature on screening (see for example [13], [14] and [15]) showed that, presently, there does not seem to exist undisputable models for screening. Consequently, no easy evaluation can be given of the screening range.

Finally it appears, as already mentioned in our previous theoretical study [1], that implementing experimentally Poole-Frenkel type approaches is not as straightforward as it is sometimes felt. 


\section{References}

[1] Ongaro R., Pillonnet A., Synthetic theory of Poole and Poole-Frenkel (PF) effects, IEE Proc. A 138 (1991) 127-37.

[2a] Mahapatra P. K., Roy C. L., Stark ladders and high-field electrical conduction in impure semiconductors, J. Phys. C: Solid State Phys. 18 (1985) 3467-81.

[2b] RoY C. L., Carrier concentrations in impure semiconductors in the presence of an electric field, $J$. Phys. Chem. Solids 47 (1986) 375-9.

[2c] RoY C. L., A note on the PF effect and some related aspects, J. Phys. Chem. Solids 47 (1986) 8256.

[3] Ongaro R., Pillonnet A., Poole-Frenkel (PF) effect hight field saturation, Rev. Phys. Appl. 24 (1989) 1085-95.

[4] VollmanN W., Poole-Frenkel conduction in insulators of large impurity densities, Phys. Stat. Sol. (a) 22 (1974) 195-203.

[5] Dussel G. A., Böer K. W., Field-enhanced ionization, Phys. Stat. Sol. 39 (1970) 375-89.

[6] Hill R. M., Poole-Frenkel conduction in amorphous solids, Philos. Mag. 23 (1971) 59-86.

[7] Hirai T., NAKada O., Formation of thin polyacrylonitrile films and their electrical properties, Japan. J. Appl. Phys. 7 (1968) 112-21.

[8] TANAKA T., INUSHI Y., High field conduction in polyethylene, Electl. Engng. Japan 89 (1969) 112.

[9] Simmons J. G., Poole-Frenkel effect and Schottky effect in metal-insulator-metal systems, Phys. Rev. 155 (1967) 657-60.

[10] Pillonnet A., Etude théorique de l'ionisation de donneurs par un champ électrique, à partir de l'effet Poole-Frenkel, Thèse, Université Lyon 1, France (1988).

[11] Lovell R., Decaying and steady currents in an epoxy polymer at high electric fields, J. Phys. D : Appl. Phys. 7 (1974) 1518-30.

[12] Ongaro R., Pillonnet A., Failure of Arrhenius plots for investigation of localized levels, Rev. Phys. Appl. 25 (1990) 209-27.

[13] Lehman G. W., James H. M., Interaction of impurities and mobile carriers in semiconductors, Phys. Rev. 100 (1955) 1698-1712.

[14] Neumark G. F., Concentration and temperature dependence of impurity-to-band activation energies, Phys. Rev. B 5 (1972) 408-17.

[15] LEE T. F., MCGILL T. C., Variation of impurity-to-band activation energies with impurity density, J. Appl. Phys. 46 (1975) 373-80. 